\title{
Assessing Data Cybersecurity Using ISO/IEC 25012
}

\author{
Javier Verdugo $^{1,2(\bowtie)}(\mathbb{D})$ and Moisés Rodríguez ${ }^{1,2}$ (1) \\ 1 AQCLab, Camino Moledores, 13071 Ciudad Real, Spain \\ \{jverdugo, mrodriguez\}@aqclab.es \\ 2 Alarcos Research Group, Institute of Technologies and Information Systems, \\ University of Castilla-La Mancha, Paseo de La Universidad, 4, \\ 13071 Ciudad Real, Spain
}

\begin{abstract}
The importance of data is ever-growing, and it is widely considered to be the most valuable asset of a company. Since data is becoming the main driver of business value, data security is a paramount concern for companies. In recent years, several standards related to security have emerged, most notably those of the ISO/IEC 27000 series. However, they are focused on management systems and security infrastructure, neglecting the security of the data itself. Other standards related to data quality, such as ISO 8000, also fail to address data security in depth. To this end, we propose in this paper a framework for the evaluation of data cybersecurity, consisting of a quality model (based on ISO/IEC 25012), an evaluation process (based on ISO/IEC 25040), and a tool for the visualization of the assessment results. This evaluation framework has been taken as the basis for a data cybersecurity certification scheme, which complements other certifiable standards related to data and security such as ISO/IEC 27001 and ISO 8000.
\end{abstract}

Keywords: Data cybersecurity $\cdot$ Quality model $\cdot$ Evaluation $\cdot$ Certification · ISO/IEC 25012

\section{Introduction}

In recent years, and due to the increasing emergence of technologies related to Open Data, Big Data, Business Intelligence, etc., data has become the most important asset of companies that blossom in the digital era. With the growing importance of data as a driver of business value, data security is becoming an even more relevant concern. An example of this is the political agreement on the Cybersecurity Act reached by the European Parliament, the Council and the European Commission. Among other things, The Cybersecurity Act creates a framework for European Cybersecurity Certificates for products, processes and services that will be valid throughout the EU [1].

However, while this EU framework for cybersecurity certification is still being implemented, the focus regarding security has so far been on infrastructure and management systems, but not on the security of the data itself. 
ISO/IEC 27000 [2] is currently the most widespread series of standards for security assessment and certification. Among the security controls proposed by the standards in this series, several are applicable to data, but the purpose of ISO/IEC 27000 is the evaluation and certification of the information security management systems (based on the Deming PDCA cycle), and it does not address specifically the evaluation and certification of data security.

Other standards related to cybersecurity focus on threats and weaknesses over software systems. An example of this is the standard Automated Source Code Security Measure (ASCSM) [3], defined by OMG, which identifies weaknesses that can be detected on the source code of applications through static analysis. As with other approaches to cybersecurity, it does not address data security specifically.

The evaluation of data cybersecurity is currently at a very early stage, and the main global reference standards that are somehow related to the subject, such as ISO/IEC 25000 [4] or ISO 8000 [5] are still under development. On the other hand, there are frameworks for the evaluation of the quality of the software product, the quality of data, the cybersecurity of critical infrastructures, and the certification of information security management systems, but none specific to data cybersecurity.

The main objective of this paper is presenting the data cybersecurity evaluation framework that has been defined, consisting of a quality model, an evaluation process, and a technological environment. The results of data cybersecurity evaluations carried out with the framework presented in this work are the basis for data cybersecurity certification, as part of the scheme defined by AENOR (leading certification body in Spain) for Cybersecurity and Privacy, which consists of several complementary certifiable standards.

The remainder of the paper is structured as follows: Sect. 2 presents the ISO/IEC 25000 series of standards, basis for the model and process proposed in this work. Section 3 presents the data cybersecurity model defined. Section 4 presents the environment for data cybersecurity evaluation and certification. Section 5 presents a case of application of data cybersecurity evaluation. Finally, Sect. 6 presents the conclusions and future work.

\section{ISO/IEC 25000 Series of Standards}

\subsection{Organization of SQuaRE Series of Standards}

ISO/IEC 25000 "Software Product Quality Requirements and Evaluation" [6], also known as SQuaRE, is a series of International Standards that stems from and revises the old standards related to software product quality, ISO/IEC 9126 [7] and ISO/IEC 14598 [8]. The ISO/IEC 25000 series was devised with the aim of amending the differences and inconsistencies between those two standards and replacing them, defining a complete framework that establishes, among others, a quality model, a set of metrics and a product quality evaluation process. The standards in the ISO/IEC 25000 family are organized in five divisions, as shown in Fig. 1. 


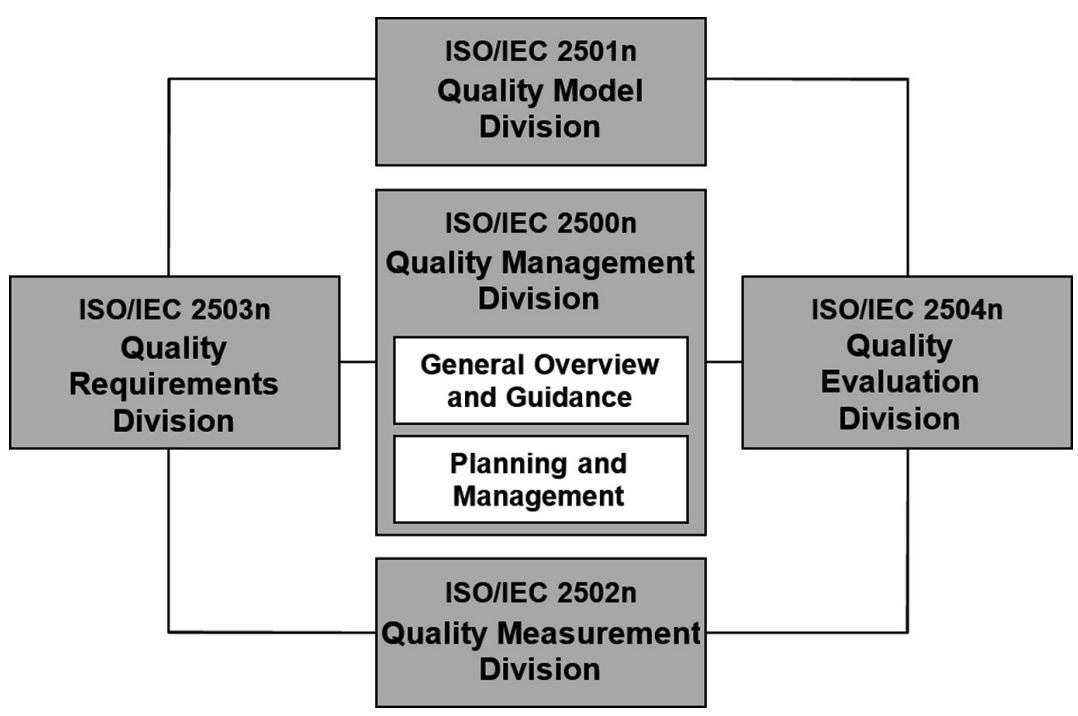

Fig. 1. Organization of the ISO/IEC 25000 series of standards (adapted from [9])

- ISO/IEC 2500n - Quality Management Division. The standards in this division define the common models, terms and definitions referred to by all the other standards in the SQuaRE series. This division also provides guidance for supporting functions responsible for the management of software product quality specification and evaluation.

- ISO/IEC 2501n - Quality Model Division. The standards in this division provide detailed models for systems and software product quality and quality in use (ISO/IEC 25010 [10]), data quality (ISO/IEC 25012 [4]), and service quality (ISO/IEC 25011 [11]). Guidance on the use of these models is provided as well.

- ISO/IEC 2502n - Quality Measurement Division. The standards that form this division include a reference model for systems and software product quality measurement (ISO/IEC 25020 [12]), definitions of quality measures for the different models in the ISO/IEC 2501n division, as well as practical guidance for their application. In this regard, ISO/IEC 25022 [13] provides measures for quality in use, ISO/IEC 25023 [14] for systems and software product quality, and ISO/IEC 25024 [15] for data quality. ISO/IEC 25025, currently under development, will provide measures for IT service quality.

- ISO/IEC 2503n - Quality Requirements Division. The standards in this division help to specify quality requirements, which can be used in the process of requirements elicitation for a system or software product to be developed, or as inputs for an evaluation process.

- ISO/IEC 2504n - Quality Evaluation Division. The standards that form this division provide a process and requirements for quality evaluation (ISO/IEC 25040 [16]), as well as recommendations and guidelines for developers, acquirers and independent evaluators). 
The numeration ISO/IEC 25050 to ISO/IEC 25099 is reserved for the extension of SQuaRe, designated to contain system or software product quality standards and/or technical reports that address specific application domains or that can be used to complement the standards in the previous five divisions. For example, ISO/IEC 25051 [17] provides requirements for quality of Ready to Use Software Product (RUSP) and instructions for their conformity evaluation, and standards in the range ISO/IEC 25060 to ISO/IEC 25066 provide a Common Industry Format (CIF) for usability-related information.

\subsection{ISO/IEC 25012 - Data Quality Model}

ISO/IEC 25012 defines a general data quality model for data that is part of a computer system. The fifteen quality characteristics defined in ISO/IEC are categorized according to two points of view:

- Inherent data quality: refers to quality characteristics that are measured on attributes of the data itself.

- System dependent data quality: refers to quality characteristics that are measured through the capabilities of the computer system retaining the data.

The classification of the fifteen data quality characteristics defined in ISO/IEC 25012 is shown in Table 1 . As the table shows, some characteristics are relevant from both points of view.

Table 1. Data quality characteristics defined in ISO/IEC 25012

\begin{tabular}{l|l|l}
\hline \multirow{2}{*}{ Characteristics } & \multicolumn{2}{|l}{ Data quality } \\
\cline { 2 - 3 } & Inherent & System dependent \\
\hline Accuracy & $\mathrm{X}$ & \\
\hline Completeness & $\mathrm{X}$ & \\
\hline Consistency & $\mathrm{X}$ & \\
\hline Credibility & $\mathrm{X}$ & \\
\hline Currentness & $\mathrm{X}$ & \\
\hline Accessibility & $\mathrm{X}$ & $\mathrm{X}$ \\
\hline Compliance & $\mathrm{X}$ & $\mathrm{X}$ \\
\hline Confidentiality & $\mathrm{X}$ & $\mathrm{X}$ \\
\hline Efficiency & $\mathrm{X}$ & $\mathrm{X}$ \\
\hline Precision & $\mathrm{X}$ & $\mathrm{X}$ \\
\hline Traceability & $\mathrm{X}$ & $\mathrm{X}$ \\
\hline Understandability & $\mathrm{X}$ & $\mathrm{X}$ \\
\hline Availability & & $\mathrm{X}$ \\
\hline Portability & & $\mathrm{X}$ \\
\hline Recoverability & & $\mathrm{X}$ \\
\hline
\end{tabular}




\subsection{ISO/IEC 25024 - Measurement of Data Quality}

ISO/IEC 25024 defines data quality measures for quantitatively measuring data quality in terms of the characteristics defined in ISO/IEC 25012 (shown in Table 1).

For each characteristic defined in ISO/IEC 25012, this standard proposes:

- A basic set of data quality measures

- A basic set of target entities to which the quality measures are applied during the data-life-cycle

- An explanation of how to apply the data quality measures

- Guidance for organizations defining their own measures for data quality

\section{Model for Data Cybersecurity Evaluation}

The data cybersecurity model presented in this work is based on the standards ISO/IEC 25012 (Data quality model) and ISO/IEC 25024 (Measurement of data quality). This model has been incorporated to the evaluation framework of AQCLab, an accredited laboratory that carries out evaluations of software product Functional Suitability [18], software product Maintainability [19] and Data Quality.

\subsection{Quality Characteristics}

The data cybersecurity model consists of a subset of five characteristics defined in the ISO/IEC 25012 data quality model, selected for their close relation to security aspects:

- Compliance: degree to which data adhere to standards, conventions, regulations and similar rules relating to data quality.

- Confidentiality: degree to which data is ensured to be only accessible and interpretable by authorized users.

- Traceability: degree to which an audit trail is provided regarding access and changes made to the data.

- Availability: degree to which data can be retrieved by authorized users and/or applications.

- Recoverability: degree to which data maintains and preserves a specified level of operations and quality, even in the event of failure.

As a result of their evaluation, each of these characteristics take a value in the scale 1 to 5 . The value in this scale represents the quality level for the characteristic, and it represents the range from deficient quality to excellent quality.

\subsection{Quality Properties}

The value of each data cybersecurity characteristic is obtained from the values of the several quality properties that intervene in their evaluation. The quality properties defined in the data cybersecurity model have been extracted from the quality measures 
proposed in ISO/IEC 25024. The specific properties for each of the characteristics of the data cybersecurity model are shown in Table 2.

Table 2. Quality properties for the evaluation of data cybersecurity characteristics and their related point of view (I: Inherent quality: S D: System dependent quality)

\begin{tabular}{|c|c|c|c|}
\hline \multirow[t]{2}{*}{ Characteristic } & \multirow[t]{2}{*}{ Properties } & \multicolumn{2}{|c|}{$\begin{array}{l}\text { Point } \\
\text { of view }\end{array}$} \\
\hline & & I & S D \\
\hline \multirow[t]{2}{*}{ Compliance } & Regulatory compliance of value and/or format & $\mathrm{X}$ & \\
\hline & Regulatory compliance due to technology & & $\mathrm{X}$ \\
\hline \multirow[t]{2}{*}{ Confidentiality } & Encryption usage & $\mathrm{X}$ & \\
\hline & Non vulnerability & & $\mathrm{X}$ \\
\hline \multirow[t]{2}{*}{ Traceability } & Users access traceability & $\mathrm{X}$ & $\mathrm{X}$ \\
\hline & Data values traceability & $\mathrm{X}$ & $\mathrm{X}$ \\
\hline \multirow[t]{3}{*}{ Availability } & Data availability ratio & & $\mathrm{X}$ \\
\hline & Probability of data available & & $\mathrm{X}$ \\
\hline & Architecture elements availability & & $\mathrm{X}$ \\
\hline \multirow[t]{3}{*}{ Recoverability } & Data recoverability ratio & & $\mathrm{X}$ \\
\hline & Periodical backup & & $\mathrm{X}$ \\
\hline & Architecture recoverability & & $\mathrm{X}$ \\
\hline
\end{tabular}

Quality properties of the data cybersecurity model take a quality value in the range $[0,100]$. Property values are taken as the basis for the evaluation of the characteristics. Thus, the value for each characteristic is obtained by applying an aggregation function over the values of its related properties. The quality value for properties is obtained by applying measurement functions over some base measures. When these base measures apply at data file level (meaning that the target entity is, for example, a table in a relational database), they are measured for each file in the data repository, and then a categorizing or profiling function is applied to obtain the property value derived from the measurements for all the files in the data repository.

The information on how to obtain the value for each property, as well as any other descriptive information necessary for their evaluation has been defined as part of the data cybersecurity model. In this manner, the model provides the following information for each property: related characteristic, property description, point of view, target entity, target attribute, measurement description, calculation formula, scale, value range, and property measurement function. Table 3 shows an example of how this information is characterized in the model, in this case, for the property Regulatory compliance of value and/or format.

Target entities for the different properties of the model are: data files (tables), elements of data architecture (contextual schema, data models, data dictionary), computer system (as a whole), and elements of system architecture (database management system, documents, forms, presentation devices). Each of these target entities have quantifiable attributes over which the base measurements for each property are defined. 
Table 3. Evaluation information for the property Regulatory compliance of value and/or format

\begin{tabular}{|c|c|c|c|}
\hline Property & \multicolumn{3}{|c|}{ Regulatory compliance of value and/or format } \\
\hline $\begin{array}{l}\text { Related } \\
\text { characteristic }\end{array}$ & \multicolumn{3}{|c|}{ Compliance } \\
\hline Description & \multicolumn{3}{|c|}{$\begin{array}{l}\text { Degree to which data values and/or format comply with spe- } \\
\text { cific standards, conventions or regulations. The organization is } \\
\text { responsible for identifying or stablishing which rules the data } \\
\text { must comply with in terms of value and/or format. Such rules } \\
\text { can be stablished, either internally by the organization owning } \\
\text { the data, or by external regulatory bodies. }\end{array}$} \\
\hline Point of view & \multicolumn{3}{|l|}{ Inherent } \\
\hline Target entity & \multicolumn{3}{|c|}{ Data file (table) } \\
\hline Target attribute & \multicolumn{3}{|c|}{ Data record (row) } \\
\hline $\begin{array}{l}\text { Measurement } \\
\text { description }\end{array}$ & \multicolumn{3}{|c|}{$\begin{array}{l}\text { Regulatory compliance of value and/or format for a data file is } \\
\text { obtained as the ratio of records of that file whose value for } \\
\text { their fields comply with specific rules, conventions or regula- } \\
\text { tions that have been established. }\end{array}$} \\
\hline $\begin{array}{l}\text { Calculation } \\
\text { formula }\end{array}$ & \multicolumn{3}{|c|}{$\begin{array}{l}\mathrm{X}=\mathrm{A} / \mathrm{B} \\
\mathrm{X}=\text { regulatory compliance of value and/or format for a file } \\
\mathrm{A}=\text { number of records that have values and/or format that } \\
\text { conform to standards, conventions or regulations } \\
\mathrm{B}=\text { number of records that shall conform to standards, conven- } \\
\text { tions or regulations due to their value }\end{array}$} \\
\hline Scale & \multicolumn{3}{|l|}{ Ratio } \\
\hline Value range & \multicolumn{3}{|c|}{$[0.0-1.0]$} \\
\hline \multirow[t]{6}{*}{$\begin{array}{l}\text { Property } \\
\text { measurement } \\
\text { function }\end{array}$} & \multicolumn{3}{|c|}{$\begin{array}{l}\text { Profile function over value of regulatory compliance of value } \\
\text { and/or format for each file. } \\
\text { Profiling criteria: }\end{array}$} \\
\hline & Level & Range & Description \\
\hline & 1 & {$[0.0-0.6)$} & $\begin{array}{l}\text { Low regulatory compliance of } \\
\text { value and/or format }\end{array}$ \\
\hline & 2 & {$[0.6-0.75)$} & $\begin{array}{l}\text { Medium regulatory compliance of } \\
\text { value and/or format }\end{array}$ \\
\hline & 3 & {$[0.75-0.95)$} & $\begin{array}{l}\text { High regulatory compliance of } \\
\text { value and/or format }\end{array}$ \\
\hline & 4 & {$[0.95-1.0]$} & $\begin{array}{l}\text { Very high regulatory compliance } \\
\text { of value and/or format }\end{array}$ \\
\hline
\end{tabular}

\section{Data Cybersecurity Environment}

In order to carry out a data cybersecurity evaluation, a methodologic and technological environment is needed. The evaluation model is the main part of the evaluation environment, but in order to be practical, it must be supported by two elements: a set of processes and activities that stablish the steps that have to be carried out and guide the 
interaction with the customers, and a set of tools to perform the measurements and evaluations and visualize the results.

Hence, the purpose of the data cybersecurity environment is setting the basis for providing companies and public organisms with data cybersecurity evaluation and certification services.

The methodologic part of the environment consists aims to define the following:

- The set of processes necessary to carry out data cybersecurity evaluations, the specific activities to be carried out, and the artifacts that are handled during the evaluation process (inputs to the process, outputs or deliverables, etc.).

- The set of processes necessary to carry out data cybersecurity certification, based on a previous evaluation.

\subsection{Evaluation Process}

The evaluation process sets the activities, inputs, outputs, resources and constraints required to carry out a data cybersecurity evaluation, in this case, from the point of view of an independent evaluator. The evaluation process defined in this work has been adapted from the process in the standard ISO/IEC 25040.

The activities and tasks of the data cybersecurity evaluation process are defined as follows. It should be taken into account that the activities are not carried out strictly one after another. Iterations on specific activities or between activities may and usually occur.

Establish the evaluation requirements. This activity consists of the following tasks:

- Establish the purpose of the evaluation: The purpose of the data cybersecurity evaluation shall be documented as a basis for the further evaluation activities and tasks.

- Obtain the data cybersecurity requirements: The stakeholders of the computer system retaining the data shall identify and provide the requirements according to the cybersecurity model defined by the evaluator. The evaluation model itself also pose a series of specific requirements that the evaluator will provide to the client in order to decide together if the evaluation method meets the client's expectations.

- Identify data and computer system parts to be included in the evaluation: All data and computer system parts to be included in the evaluation shall be identified and documented.

Specify the evaluation. This activity consists of the following tasks:

- Select quality measures (evaluation modules): The evaluator shall select quality measures (evaluation modules) to cover all data cybersecurity evaluation requirements. Since the measures of the model are pre-stablished, in this activity the evaluator identifies the mapping between the measures, the data and computer system parts, and the data cybersecurity requirements.

- Define decision criteria for quality measures: Decision criteria (numerical thresholds and targets) for the individual measures is already defined as part of the evaluation model. 
- Define decision criteria for evaluation: The decision criteria for the quality characteristics, whose value is obtained from the combination of values for quality properties, already defined as part of the evaluation model.

Design the evaluation. This activity consists of the following task:

- Plan evaluation activities: The evaluation activities shall be scheduled, taking into account the availability of resources and the data and computer system parts to be evaluated.

Execute the evaluation. This activity consists of the following tasks:

- Make measurements: The data cybersecurity measures shall be applied to the data and computer system parts, according to the evaluation plan, resulting in values on the measurement scales.

- Apply decision criteria for quality measures: The decision criteria for the measures shall be applied to the measured values, obtaining values for the quality properties. This activity is automated thanks to the technological framework available.

- Apply decision criteria for evaluation: The set of decision criteria shall be applied to the characteristics, parting from the property values, and producing the evaluation results.

Conclude the evaluation. This activity consists of the following tasks:

- Review the evaluation result: The evaluator and the client shall carry out a joint review of the evaluation results.

- Create the evaluation report: The evaluator shall create the evaluation report, detailing the results, and including descriptive information of the evaluation (requirements, evaluation modules, plan, personnel, etc.). The evaluator shall provide the client with the report created.

- Review quality evaluation and provide feedback to the organization: The evaluator shall review the results of the evaluation. Feedback from the review should be used in order to improve the evaluation process and evaluation techniques (evaluation modules).

- Perform disposition of evaluation data: When the evaluation is completed the data and any other items that may have been provided by the client shall be disposed.

\subsection{Certification Process}

The data cybersecurity evaluation framework described in this work has been taken as the basis by the Spanish leading certification body, AENOR, for their data cybersecurity certification scheme, which is part of their certification portfolio for Cybersecurity and Privacy, along with other certifiable standards.

The process for obtaining a data cybersecurity certificate consists of the six interaction steps between client, evaluator and certification body shown in Fig. 2. 
(1) Data Cibersecurity Evaluation Request

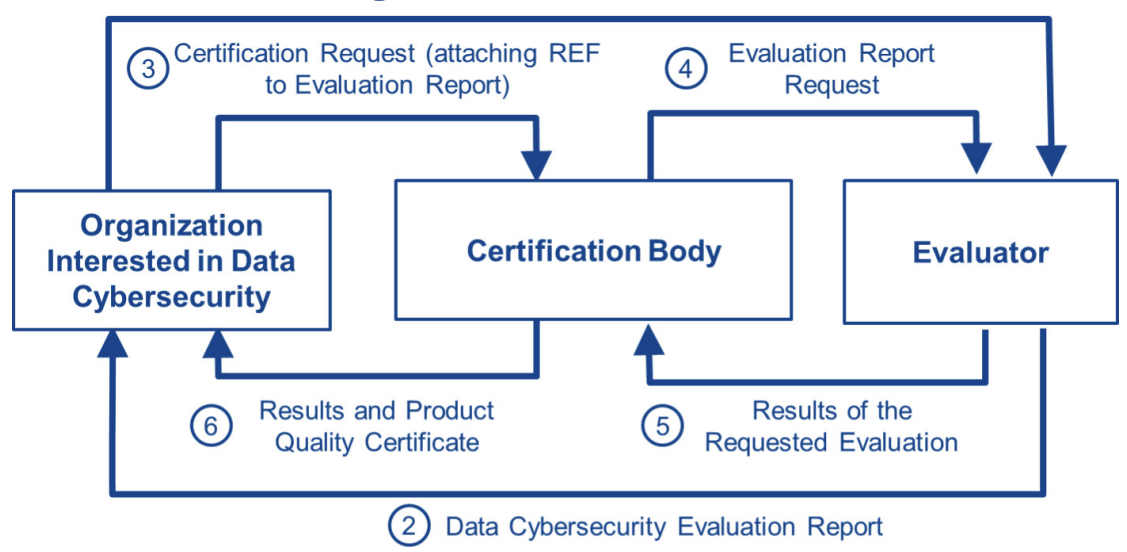

Fig. 2. Steps of the data cybersecurity certification process

\subsection{Technological Environment}

A technological environment is necessary so that the evaluation of data cybersecurity can be carried out in a practical, efficient and accurate way. This technological environment consists of tools that automate the acquisition, calculation and presentation of the values obtained for the characteristics, properties and measures defined in the evaluation model.

The measurement tools used in the evaluation to obtain the values for base measures depend vastly on the technologies of the computer system of the data product evaluated. For example, for relational databases a query tool can be used to communicate with the DBMS and obtain information needed for some measures of the model.

The evaluation tool of the environment is responsible for applying the decision criteria of the evaluation model. For that, it takes as input the measurement of the base measures defined in the model, once they have been obtained from the target entities. The evaluation tool takes the base measure values specified in an XML file and processes them to obtain the values for quality properties. This processing consists in applying evaluation functions that represent the decision criteria defined in the model as thresholds and profiles. The values for the quality properties are in turned processed to obtain the values for the characteristics of the model. The resulting values for the properties and characteristics after the evaluation are stored in a database.

A visualization tool has been developed in order to consult and represent the results of data cybersecurity evaluations in a clear and concise way. This tool is aimed to be used by both evaluators and clients. The permissions system of the tool allows to control which functionalities and information of which evaluations the user can access based on her role. When the user selects an evaluation (between the ones available to her), its information is displayed (see Fig. 3): name of the data product, version, date of the evaluation, value for characteristics and properties in tabular way, and graphics representing those values (radar chart for characteristics, bar chart for properties). 


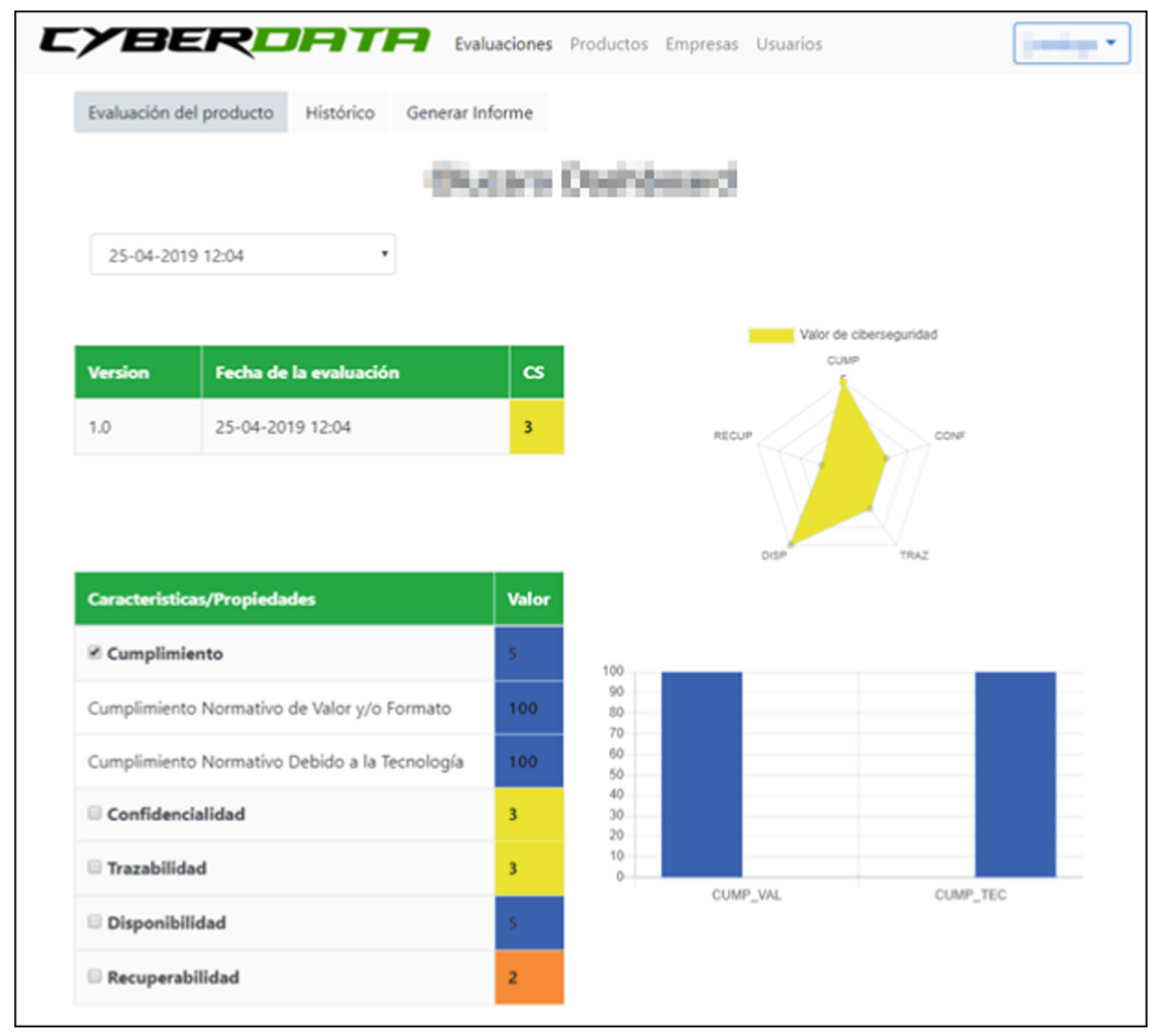

Fig. 3. Snapshot of the visualization tool for data cybersecurity evaluations

The visualization tool provides more functionalities, such as generating downloadable reports for evaluations, showing trends in the values of cybersecurity evaluations, managing companies, data products and users, etc.

\section{Example of Application}

As a pilot project, the evaluation framework presented in this work has been applied to a commercial product: a business dashboard management tool provided to its users under a SaaS model. This tool uses the balanced scorecard (BSC) approach, allowing the users to define, monitor and control their own KPIs. The evaluation was conducted following the process and using the technological environment defined in the previous section, and applying the model defined in Sect. 3.

As a result of the first activity, Establish the evaluation requirements, the cybersecurity model was presented to the client, explaining the characteristics and properties, and the generic requirements related to their measures. The client identified the data and elements of the computer system in the scope of the evaluation: 
- The database of the application, being all the tables part of the target for the evaluation.

- The application itself, hosted in a server managed by the client.

- The DBMS of the application.

- The latest backup copies made.

- The results of penetration testing carried out.

- Documentation of the application and computer system.

Specific requirements for some characteristics were also identified by the client, such as rules regarding value or format for some data fields, fields that require to contain encrypted data with a specific algorithm, the frequency of the backups, etc.

As a result of the second activity, Specify the evaluation, since the measures for the evaluation were already defined, the mapping between the measures, the data and computer system parts, and specific requirements was carried out.

The third activity, Specify the evaluation, resulted in the evaluation plan, with the allocation of resources to activities.

Then, the fourth activity, Execute the evaluation, was carried out. The base measurements were performed over the target entities according to their specification in the model and the specific requirements identified by the client for some of the quality properties. The results of the measurement were processed with the evaluation tool in order to obtain the values for properties and characteristics. The results obtained are shown in Table 4.

Table 4. Quality values for the data cybersecurity characteristics obtained in the evaluation

\begin{tabular}{l|l}
\hline Characteristic & Value \\
\hline Compliance & 5 \\
\hline Confidentiality & 3 \\
\hline Traceability & 3 \\
\hline Availability & 5 \\
\hline Recoverability & 2 \\
\hline
\end{tabular}

To finish the evaluation, the last activity, Conclude the evaluation, was carried out. First, the results of the evaluation were reviewed, and then the evaluation report was generated with the visualization tool and issued to the client.

\section{Conclusions and Future Work}

This work presents a first step in a domain that is becoming more and more important, but has not been explored in depth. Although there are many approaches to data quality and cybersecurity, such as standards, models, best practices, etc. none of them actually take into account both at the same time. Data quality approaches usually do not address security aspects comprehensively, and cybersecurity approaches usually focus more on systems infrastructure, software, and networks than on the data. 
This work has presented an industrial approach to data cybersecurity based on International Standards. The pilot project conducted has shown that the proposed framework can be used to evaluate and certificate the cybersecurity of the data that companies manage and work with as part of their business mission.

In the future we intend to carry out more evaluations with this framework in order to get more practical knowledge about the feasibility of this type of evaluations, the drawbacks regarding the measures selected and how to apply them in real-life information systems, and in general, make improvements on the data cybersecurity evaluation framework proposed so that it addresses the needs of the market.

Acknowledgements. This research is part of the DQIoT project (INNO-20171086), funded by CDTI; ECD project (PTQ-16-08504), funded by the "Torres Quevedo" Program of the Spanish Ministry of Economy, Industry and Competitiveness; CYBERDATA project (REF: 13/17/IN/013) funded by Consejería de Economía, Empresas y Empleo JCCM and FEDER (Fondo Europeo de Desarrollo Regional); ECLIPSE project (Ministerio de Ciencia, Innovación y Universidades, and Fondo Europeo de Desarrollo Regional FEDER, RTI2018-094283-B-C31); and TESTIMO project (Consejería de Educación, Cultura y Deportes de la Junta de Comunidades de Castilla La Mancha, and Fondo Europeo de Desarrollo Regional FEDER, SBPLY/17/180501/000503).

\section{References}

1. European Comission. https://ec.europa.eu/commission/news/cybersecurity-act-2018-dec-11_ en. Accessed 16 May 2019

2. ISO/IEC 27000: Information technology - Security techniques - Information security management systems - Overview and vocabulary. International Organization for Standardization/ISO/IEC JTC 1/SC 27 Information Security, cybersecurity and privacy protection (2018)

3. ASCSM 1.0: Automated Source Code CISQ Security Measure. Object Management Group (2016)

4. ISO/IEC 25012: Software Engineering - Software product Quality Requirements and Evaluation (SQuaRE) - Data Quality Model. International Organization for Standardization/ ISO/IEC JTC 1/SC 7 Software and systems engineering (2008)

5. ISO/TS 8000-60: Data Quality - Part 60: Data Quality Management: Overview. International Organization for Standardization/TC 184/SC 4 Industrial data (2017)

6. ISO/IEC 25000: Systems and software engineering - Systems and software Quality Requirements and Evaluation (SQuaRE) - Guide to SQuaRE. International Organization for Standardization/ISO/IEC JTC 1/SC 7 Software and systems engineering (2014)

7. ISO/IEC 9126-1: Software engineering - Product quality - Part 1: Quality model. International Organization for Standardization/ISO/IEC JTC 1/SC 7 Software and systems engineering (2001)

8. ISO/IEC 14598-1: Information technology - Software product evaluation - Part 1: General overview. International Organization for Standardization/ISO/IEC JTC 1/SC 7 Software and systems engineering (1999)

9. Zubrow, D.: Measuring Software Product Quality: the ISO 25000 Series and CMMI. SEI (2004) 
10. ISO/IEC 25010: Software Engineering - Software product Quality Requirements and Evaluation (SQuaRE) - System and software quality models. International Organization for Standardization/ISO/IEC JTC 1/SC 7 Software and systems engineering (2011)

11. ISO/IEC TS 25011: Software Engineering - Software product Quality Requirements and Evaluation (SQuaRE) - Service quality models. International Organization for Standardization/ISO/IEC JTC 1/SC 7 Software and systems engineering (2017)

12. ISO/IEC 25020: Software Engineering - Software product Quality Requirements and Evaluation (SQuaRE) - Measurement reference model and guide. International Organization for Standardization/ISO/IEC JTC 1/SC 7 Software and systems engineering (2007)

13. ISO/IEC 25022: Software Engineering - Software product Quality Requirements and Evaluation (SQuaRE) - Measurement of quality in use. International Organization for Standardization/ISO/IEC JTC 1/SC 7 Software and systems engineering (2016)

14. ISO/IEC 25023: Software Engineering - Software product Quality Requirements and Evaluation (SQuaRE) - Measurement of system and software product quality. International Organization for Standardization/ISO/IEC JTC 1/SC 7 Software and systems engineering (2016)

15. ISO/IEC 25024: Software Engineering - Software product Quality Requirements and Evaluation (SQuaRE) - Measurement of data quality. International Organization for Standardization/ISO/IEC JTC 1/SC 7 Software and systems engineering (2015)

16. ISO/IEC 25040: Software Engineering - Software product Quality Requirements and Evaluation (SQuaRE) - Evaluation process. International Organization for Standardization/ ISO/IEC JTC 1/SC 7 Software and systems engineering (2011)

17. ISO/IEC 25051: Software Engineering - Software product Quality Requirements and Evaluation (SQuaRE) - Requirements for quality of Ready to Use Software Product (RUSP) and instructions for testing. International Organization for Standardization/ISO/IEC JTC 1/SC 7 Software and systems engineering (2014)

18. Rodríguez, M., Oviedo, J.R., Piattini, M.: Evaluation of software product functional suitability: a case study. Softw. Qual. Prof. 18(3), 18-29 (2016)

19. Rodríguez, M., Piattini, M., Fernandez, C.M.: A hard look at software quality: Pilot program uses ISO/IEC 25000 family to evaluate, improve and certify software products. Qual. Prog. 48, 30-36 (2015) 